\title{
Embedded Solitons in Lagrangian and Semi-Lagrangian Systems
}

\author{
D. J. Kaup ${ }^{1}$ and Boris A. Malomed ${ }^{1,2}$ \\ ${ }^{1}$ Department of Mathematics \\ University of Central Florida \\ Orlando, FL 32816, USA \\ kaup@ucf.edu \\ ${ }^{2}$ Department of Interdisciplinary Studies \\ Faculty of Engineering \\ Tel Aviv University \\ malomed@eng.tau.ac.il
}

\begin{abstract}
We develop the technique of the variational approximation for solitons in two directions. First, one may have a physical model which does not admit the usual Lagrangian representation, as some terms can be discarded for various reasons. For instance, the second-harmonic-generation (SHG) model considered here, which includes the Kerr nonlinearity, lacks the usual Lagrangian representation if one ignores the Kerr nonlinearity of the second harmonic, as compared to that of the fundamental. However, we show that, with a natural modification, one may still apply the variational approximation (VA) to those seemingly flawed systems as efficiently as it applies to their fully Lagrangian counterparts. We call such models, that do not admit the usual Lagrangian representation, semi-Lagrangian systems. Second, we show that, upon adding an infinitesimal tail that does not vanish at infinity, to a usual soliton ansatz, one can obtain an analytical criterion which (within the framework of VA) gives a condition for finding embedded solitons, i.e., isolated truly localized solutions existing inside the continuous spectrum of the radiation modes. The criterion takes a form of orthogonality of the radiation mode in the infinite tail to the soliton core. To test the criterion, we have applied it to both the semi-Lagrangian truncated version of the SHG model and to the same model in its full form. In the former case, the criterion (combined with VA for the soliton proper) yields an exact solution for the embedded soliton. In the latter case, the criterion selects the embedded soliton with a relative error $\approx 1 \%$.
\end{abstract}




\section{Introduction}

One of the many pioneer contributions by A.C. Newell has been the study of solitary waves (which we will simply call "solitons" here, in line with currently adopted terminology). These occur not only in integrable models, but also in many nonintegrable nonlinear-wave systems [1, 2]. It is toward an improved understanding of solitons in nonintegrable systems, that we present this work, dedicated to Prof. Newell.

In nonintegrable models, more complex forms of solitons are found as one considers higher-order systems. An issue of fundamental importance is to find where, in the space of the soliton parameters, such solutions could exist. Where this could be is dominantly determined by the model's linear dispersion relation, $\omega(k)$. Typically, dispersion curves have gaps or partial gaps, which are values of $\omega$ at which $k$ would take imaginary or complex values. For example, for the nonlinear Schrödinger (NLS) equation, one has $\omega=D k^{2}$, where $D$ is a positive dispersion coefficient, hence $k$ must be imaginary if $\omega<0$. This is precisely the region where one finds solitons of the NLS equation. For more complex systems, with several branches of the dispersion relation, one can have regions of $\omega$ where $k$ can be complex as well as real. Here one typically encounters "delocalized solitons" (see the book [3] and relevant examples from nonlinear-optical models in Refs. [4]), which are quasi-solitary waves with nonvanishing oscillatory tails. Such objects, obviously, have an infinite energy, and therefore are unphysical except, possibly, in finite-size systems. However, it may occur that the amplitude of the tail vanishes at some special values of $\omega$. Then, one has a truly localized object at an isolated (discrete) value of $\omega$ at which a real value of $k$ does exist. Since this value of $\omega$ lies in a continuous part of the spectrum, these objects are called embedded solitons (ESs). A number of examples of ESs in physically meaningful models are now known (see examples of such solitons found in the hydrodynamical models in Refs. [5], and a short review in Ref. [6]), their stability (which turns out to be semi-stability) being quite distinct from the stability of ordinary solitary waves [7].

Currently, the only method for locating ESs is to search for them numerically. One does know that they may be found inside a partial gap of the dispersion relation, but otherwise one has no analytical tool for locating them (in exceptional cases, exact ES solutions can be found by guess [7]). The first objective of this paper is to develop an approximate analytical method for locating ESs. The method is based on the variational approximation (VA; see a recent review of the application of this technique to solitons in Ref. [8]), which incorporates an amplitude of the infinitesimal tail of the related delocalized soliton as a key variational parameter. The approach will be tested on two versions of an ES-generating model introduced in Ref. [7], which combines the second-harmonic generation (SHG) through quadratic $\left[\chi^{(2)}\right]$ nonlinearity, and the usual cubic $\left[\chi^{(3)}\right]$ nonlinear terms. The difference between the two versions of the model is that one is a full model (it has the usual Lagrangian representation), in terms of the $\chi^{(3)}$ terms, while the one model is a truncated model, wherein, upon assuming that the fundamental-harmonic (FH) field is much stronger than the second harmonic $(\mathrm{SH})$, some of the $\mathrm{SH} \chi^{(3)}$ terms are omitted. The truncated model does not admit the usual Lagrangian representation. However, it was this truncated version of the $\chi^{(2)}: \chi^{(3)}$ model in which the above-mentioned exact analytic ES solution was found in Ref. [7]. On the other hand, in the full version of the model, ES solutions could only be found by means of numerical methods. We will obtain a natural variational criterion which makes it possible to distinguish ESs from delocalized solitons in each of these systems. Furthermore, we will demonstrate that, in the case of the truncated model, this method yields an exact result, and in the full model, a relative error is $\approx 1 \%$ in the prediction of the location of ES (in comparison with numerical results).

A distinctive feature of the truncated model is that, due to a missing (omitted) term, it does not have a "complete" Lagrangian representation. Namely, it can be obtained from a Lagrangian, but 
only if one term in it is not subjected to the variation with respect to the $\mathrm{FH}$ field, when deriving the system of the $\mathrm{FH}$ and $\mathrm{SH}$ equations. Accordingly, the truncated model does not conserve any Hamiltonian. But it is, nevertheless, a conservative system, as it conserves the norm of the solution (which is usually called "energy" in nonlinear optics and is different from the Hamiltonian).

As the truncated model does not admit the full Lagrangian representation, it is necessary to work out a special version of the VA for it, which is another objective of this paper. In fact, this can be done in a very simple way: after inserting the variational ansatz into the Lagrangian and performing the integration in order to obtain the corresponding effective Lagrangian (the one which is an explicit function of variational parameters, rather than a functional depending on the field variables), the above-mentioned special term in the effective Lagrangian should not be varied with respect to variational parameters that belong to the $\mathrm{FH}$ component of the ansatz. Thus, other models similar to the above-mentioned truncated one, even though they cannot be represented in the usual Lagrangian form, can still be handled by means of VA. Since one cannot freely vary all the fields in the Lagrangian of these systems, we call them semi-Lagrangian systems.

The rest of the paper is organized as follows. In section 2, we introduce the full and truncated models and the Lagrangian representations of each. In section 3, the VA for the semi-Lagrangian case is developed. The general VA-based analytical condition for identifying ESs (in an approximate form) is obtained in section 4 . Section 5 concludes the paper.

\section{The full and truncated models}

Following Refs. [9] and [7], we first introduce the full $\chi^{(2)}: \chi^{(3)}$ model:

$$
\begin{gathered}
i u_{z}+(1 / 2) u_{t t}+u^{*} v+\gamma_{1}\left(|u|^{2}+2|v|^{2}\right) u=0, \\
i v_{z}-(1 / 2) \delta v_{t t}+q v+(1 / 2) u^{2}+2 \gamma_{2}\left(|v|^{2}+2|u|^{2}\right) v=0,
\end{gathered}
$$

which is written in the usual "optical" notation, so that $z$ and $t$ are the propagation distance and reduced time, $u$ and $v$ are the $\mathrm{FH}$ and $\mathrm{SH}$ fields, $-\delta$ is the relative dispersion coefficient at $\mathrm{SH}, q$ is the SHG mismatch, and $\gamma_{1,2}$ are Kerr coefficients. In fact, the ratio of the self-phase-modulation (SPM) and cross-phase-modulation (XPM) coefficients is not necessarily $1: 2$ simultaneously in both equations, as it is written in Eqs. (1) and (2), but this feature of the model is not a crucially important one.

The form of Eqs. (11) and (2) implies that the group-velocity dispersion is anomalous at $\mathrm{FH}$, while at SH it may be both normal, if $\delta>0$, and anomalous, if $\delta<0$ (both cases are physically possible). As for the Kerr coefficients $\gamma_{1}$ and $\gamma_{2}$, they always have one sign. Most typically, they are positive (corresponding to the self-focusing nonlinearity), but may be negative too, see a detailed discussion of this point in Ref. [10].

In many cases, the $\mathrm{SH}$ field is much weaker than the $\mathrm{FH}$ field - for instance, if the mismatch is large. Then, assuming that $|v|^{2} \ll|u|^{2}$, one may neglect the XPM term in comparison with the SPM one in Eq. (1), and the SPM term in comparison with its XPM counterpart in Eq. (2), which leads to the truncated model,

$$
\begin{gathered}
i u_{z}+(1 / 2) u_{t t}+u^{*} v+\gamma_{1}|u|^{2} u=0 \\
i v_{z}-(1 / 2) \delta v_{t t}+q v+(1 / 2) u^{2}+4 \gamma_{2}|u|^{2} v=0 .
\end{gathered}
$$


In this work, we are interested in stationary fundamental-soliton solutions, which are looked in the form

$$
u(z, t)=e^{i k z} U(t), v(z, t)=e^{2 i k z} V(t),
$$

where $k$ is the $\mathrm{FH}$ wavenumber, and real even functions $U(t)$ and $V(t)$ with a single maximum at $t=0$ exponentially decay at $t \rightarrow \infty$. Note that ordinary (non-embedded) solitons may exist in the regions

$$
0<k<q / 2 \text {, if } \delta>0 ; k>\max \{0, q / 2\} \text {, if } \delta<0
$$

(which implies that $q$ must be positive for the existence of ordinary solitons if $\delta$ is positive). On the other hand, ESs may exist in a range of $k$ which does not overlap with the continuous spectrum in the FH equation, (11) or (3), but falls into the continuous spectrum of the SH equation, (2) or (4). Thus, ES may exist in the regions [cf. Eqs. (6)]

$$
k>\max \{0, q / 2\}, \text { if } \delta>0 ; 0<k<q / 2 \text {, if } \delta<0 .
$$

This implies that, in the case $\delta<0$, the existence of ESs makes it necessary to have positive $q$.

Substituting the expressions (5) into Eqs. (11) and (2), we arrive at a system of ordinary differential equations,

$$
\begin{gathered}
-k U+(1 / 2) U^{\prime \prime}+U^{*} V+\gamma_{1}\left(U^{2}+2 V^{2}\right) U=0, \\
-2 k V-(1 / 2) \delta V^{\prime \prime}+q v+(1 / 2) U^{2}+2 \gamma_{2}\left(V^{2}+2 U^{2}\right) V=0,
\end{gathered}
$$

where the prime stands for $d / d t$. Accordingly, the system of stationary equations corresponding to the truncated model is

$$
\begin{gathered}
-k U+(1 / 2) U^{\prime \prime}+U^{*} V+\gamma_{1} U^{3}=0, \\
-2 k V-(1 / 2) \delta V^{\prime \prime}+q V+(1 / 2) U^{2}+4 \gamma_{2} U^{2} V=0 .
\end{gathered}
$$

It is obvious that Eqs. (8) and (9) can be derived from the Lagrangian

$$
L=\frac{1}{2} \int_{-\infty}^{+\infty}\left[-k U^{2}-(2 k+q) V^{2}-\frac{1}{2}\left(U^{\prime}\right)^{2}+\frac{\delta}{2}\left(V^{\prime}\right)^{2}+U^{2} V+\frac{\gamma_{1}}{2} U^{4}+4 \gamma_{2} U^{2} V^{2}+\frac{\gamma_{2}}{2} V^{4}\right] d t .
$$

In the case of the truncated system of stationary equations, (10) and (11), one may still obtain this system from the variational principle, provided we do the following. First, the last term in the integrand in Eq. (12) should be dropped. Second, the next to the last term should only be subjected to varying in $V$, but not in the $U$ field.

\section{The variational approximation for the semi-Lagrangian system}

In this section, we focus on the application of VA to the semi-Lagrangian (truncated) system (10), (11). The most natural variational ansatz to search for embedded solitons proper (without the tail, which will be considered in the next section) in this system is based on the following expressions:

$$
U=A \operatorname{sech}(\sqrt{2 k} x) x, V=B \operatorname{sech}^{2}(\sqrt{2 k} x),
$$


where the amplitudes $A$ and $B$ are variational parameters, while the inverse width $\sqrt{2 k}$ has been fixed to match to the linearized form of Eqs. (8) and (9) or (10) and (11) at $t \rightarrow \infty$. Substituting the ansatz (13) into the Lagrangian (12), dropping the last term, and then performing the integration, we find the effective Lagrangian of the truncated system:

$$
3 \sqrt{2 k} L_{\text {eff }}=-4 k A^{2}-2\left[2\left(1-\frac{2 \delta}{5}\right) k-q\right] B^{2}+2 A^{2} B+\gamma_{1} A^{4}+\frac{35}{5} \gamma_{2} A^{2} B^{2} .
$$

As it was said above, in order to go from the full system to its truncated counterpart, one not only has to omit the last term in Eq. (12), but also must take care to not vary the term $4 \gamma_{2} U^{2} V^{2}$ with respect to $U$. In terms of the effective Lagrangian (14), this means that, when deriving the variational equations for $A$ and $B$, one should not vary the last term of the effective Lagrangian with respect to $A$. In this case, the variation with respect to $A$ yields a simple equation which allows one to eliminate $B$,

$$
B=-\gamma_{1} A^{2}+2 k
$$

Using this result, the equation produced by varying the Lagrangian with respect to $B$ can be cast into the final form of a biquadratic equation for $A$,

$$
\frac{32}{5} \gamma_{1} \gamma_{2} A^{4}-\left[1+\frac{64}{5} k \gamma_{2}+2 \gamma_{1}\left(2\left(1-\frac{2 \delta}{5}\right) k-q\right)\right] A^{2}+4 k\left[2\left(1-\frac{2 \delta}{5}\right) k-q\right]=0 .
$$

Thus, depending on values of the parameters, Eq. (16) may give up to two different physical solutions. The simplest nontrivial case (which still allows an ES to exist) is with $\gamma_{1}=0$, but $\gamma_{2} \neq 0$. Then, Eq. (16) yields a single solution,

$$
A^{2}=\frac{4 k[2(5-2 \delta) k-5 q]}{5+64 k \gamma_{2}}
$$

which is physical if it gives $A^{2}>0$; the amplitude $B$ can then be obtained from Eq. (15).

It should be stressed that, as it was demonstrated in Ref. [7], the ansatz (13) yields exact soliton solutions to Eqs. (10) and (11) at some uniquely selected value of $k$ [an expression for it is given below in Eq. (25)]. Depending on the value of the mismatch parameter $q$, this exact solution may be either ES or an ordinary soliton. Comparing Eqs. (15) and (16) with that solution, one can easily verify that expressions (15) and (16) are precisely parts of the exact solution.

Although the exact solution for the ES in the truncated model is available at the single value of $k$ at which it may exist, in the region (6), where a continuous family of ordinary solitons is expected to exist, no general exact solution is known. So, to illustrate the accuracy and reliability of the modified VA for producing approximate solutions to the truncated equations (10) and (11), in Fig. 1 we display a typical example of a numerically found ordinary soliton in the region $0<k<q / 2$ (in this example, $\delta=1$ ), together with the analytical approximation generated by Eqs. (13), (15), and (16). As one looks at the numerical solution in Fig. 1, one observes that the SH component goes slightly negative along the shoulder of the soliton, and then appears to oscillate as it decays. Note that in the region where this behavior of the $\mathrm{SH}$ component is observed, $U$ is not small, and, according to Eq. (14), the solution for $V$ in this region should oscillate indeed. Thus, ordinary solitons in the truncated system may have fine features which are not found in the ESs.

\section{An analytical criterion to identify embedded solitons}




\subsection{General analysis}

In the case when the wavenumber $k$ falls into the regions (7), ESs can exist in both the full and truncated models, i.e., (17), (2) and (3), (四) [7]. However, except for using the exact ES solutions in the truncated model to guess where ESs might exist in its full counterpart, the only known method for locating ESs in the full model was to search for them by means of direct numerical computations.

Thus, there is a need for an analytical approach to the quest for ESs. Such an approach can be based on VA, if one assumes that, at values of $k$ close to $k_{\mathrm{ES}}$, at which an ES exists, there also exists a family of delocalized solitons, with small-amplitude oscillating tails in the SH component that vanish when $k$ become equal to $k_{\mathrm{ESS}}$. As it immediately follows from Eqs. (9) or (11), the free oscillating tail of a delocalized soliton, which has an infinitesimal amplitude $b$ and arbitrary phase shift $\psi$, is given by the expression

$$
V_{\text {tail }}=b \cos (\sqrt{(2 / \delta)(2 k-q)} t+\psi),
$$

that neglects the nonlinear terms. Now, one may add this tail to the ansatz (13), to have a more general tailed ansatz,

$$
V(t)=V_{\mathrm{sol}}(t)+b \cos (\sqrt{(2 / \delta)(2 k-q)} t+\psi)
$$

where $V_{\text {sol }}(t)$ corresponds to the ansatz for the core of the delocalized soliton.

As $b$ is an extra variational parameter, one should add the variational equation

$$
\partial L_{\mathrm{eff}} / \partial b=0
$$

to the set of equations obtained by varying the effective Lagrangian with respect to all the other free parameters (irrespective of the fact if the system is complete Lagrangian or semi-Lagrangian). As we are interested in the location of an ES which, by itself, has $b=0$ (no tail), one should set $b=0$ after completing the differentiation in Eq. (20). This means that, prior to varying in $b$, one should only keep terms in $L_{\text {eff }}$ which are linear in $b$, hence Eq. (20) takes the general form,

$$
\int_{-\infty}^{+\infty}\left[\left.\left(\frac{\delta L}{\delta V}\right)\right|_{U=U_{\mathrm{sol}}(t), V=V_{\mathrm{sol}}(t)}\right] \cos (\sqrt{(2 / \delta)(2 k-q)} t+\psi) d t=0
$$

with $\delta / \delta V$ standing for the variational derivative of the underlying Lagrangian [the one given by Eq. (12)].

Let us make a couple of observations here. First, the above applies equally well to the full system as well as to the truncated system, since only variations in the SH field are involved, while the differences between the two types of systems involve solely the variation in $U$. Furthermore, since the solitons sought for are even, the expression $\delta L / \delta V$ with $V$ substituted by $V_{\text {sol }}(t)$ is also even. Hence Eq. (21) amounts to an orthogonality condition between the infinitesimal tail and the soliton,

$$
\int_{-\infty}^{+\infty}\left[\left.\left(\frac{\delta L}{\delta V}\right)\right|_{U=U_{\mathrm{sol}}(t), V=V_{\mathrm{sol}}(t)}\right] \cos (\sqrt{(2 / \delta)(2 k-q)} t) d t=0 .
$$

This consideration also shows that the phase parameter $\psi$ in the expression (18) is not important in the limit of $b=0$.

We notice that the above derivation circumvents the formal problem of the divergence of the integral expression (12) for the Lagrangian, when there is a tail which does not vanish as $|t| \rightarrow \infty$. 
The divergence did not appear since the tail was designed as a solution to the linearized version of Eqs. (9) or (11). One can readily verify that the divergence can also be eliminated in the fully nonlinear case, with respect to the tail (provided one suitably adjusts the frequency of the tail according to the nonlinearity).

Thus, Eq. (22) is a general criterion that can be used to locate ES solutions within the framework of VA. In Eq. (22), we recognize that the variational derivative $\left.(\delta L / \delta V)\right|_{U=U_{\mathrm{sol}}, V=V_{\mathrm{sol}}(t)}$ is just the left-hand side (l.h.s.) of the stationary equation for SH, with $U$ and $V$ taken as per the chosen ansatz for the core of the delocalized soliton. Another point is that upon applying integration by parts to the second-derivative term in Eq. (11), one sees that all contributions from the linear terms cancel in Eq. (22), leaving only the nonlinear terms in Eq. (11) to determine this condition.

\subsection{Embedded soliton in the truncated system}

To test the efficiency of the criterion (22), we first apply it to the truncated system. Then, the variational derivative in Eq. (22) is replaced by the nonlinear part of l.h.s. of Eq. (11),

$$
\int_{-\infty}^{+\infty}\left[\frac{1}{2} U^{2}(t)+4 \gamma_{2} U^{2}(t) V(t)\right] \cos (\sqrt{(2 / \delta)(2 k-q)} t) d t=0
$$

Using the ansatz (13), it is easy to explicitly perform the integration in Eq. (23), which finally yields a simple result (note that the $\mathrm{FH}$ amplitude drops out),

$$
4 \gamma_{2} B=-\frac{3 \delta \cdot k}{2 k(1+2 \delta)-q} .
$$

Now, combining the above results (16) and (15), which were obtained by means of the VA, with the relation (24) that locates where the ES must be (and is also based on VA), one can easily verify that this set of three relations is precisely tantamount to the exact analytical solution for the ES which was found by guess in Ref. [7]. In particular, an eventual expression for the wavenumber of ES is

$$
k_{\mathrm{ES}}=\frac{1}{2}(1+2 \delta)^{-1}\left[q-\frac{3}{2} \delta\left(4 \gamma_{2}+3 \delta \gamma_{1}\right)^{-1}\right]
$$

The fact that, in the case of the semi-Lagrangian truncated system, VA reproduces the exact ES is remarkable, although the reason for this occurrence is not fully understood. We also note that this exact soliton is not always an embedded one, as the wavenumber (25) is not necessarily restricted to the region (7) in which ES may exist: the wavenumber may instead fall into the region (6), in which ordinary solitons are to be found. A condition (inequality) showing whether the exact soliton is embedded or ordinary has already been given in Ref. [7].

\subsection{Embedded soliton in the full system}

The next step is to apply the general ES-selecting criterion (22) to the full system which is based on Eqs. (8) and (9). To this end, we assume that the soliton proper may again be approximated by the ansatz (13). Then, substituting the nonlinear part of l.h.s. of Eq. (9) into Eq. (22), we arrive, instead of Eq. (24), at a more complicated relation,

$$
4 \gamma_{2} B\left[1+\frac{B^{2}}{A^{2}} \frac{2(8 \delta+1) k_{\mathrm{ES}}-q}{40 \delta \cdot k_{\mathrm{ES}}}\right]=-\frac{3 \delta \cdot k_{\mathrm{ES}}}{2 k_{\mathrm{ES}}(1+2 \delta)-q} .
$$


We test the validity of this relation in the following way: take a particular example of the ES in the full system that was found in a numerical form in Ref. [7], for which $\delta=1, q=1$, and $\gamma_{1}=\gamma_{2}=-0.05$. Borrowing values of the amplitudes $A$ and $B$ directly from the numerical data, we find that $A=3.794$ and $B=2.735$. Substituting these values into Eq. (26) yields $k_{\mathrm{ES}}=0.688$, while the numerical value found in Ref. [7] was $k_{\mathrm{ES}}=0.696$. Thus, the relative error of the criterion (22) for this case is $1.1 \%$.

\section{Conclusion}

In this work, we have put forward two modifications to the technique of the variational approximation for solitons. First, it may happen that a physical model does not admit the full Lagrangian representation, as some terms may be missing due to various reasons. In the case of the $\chi^{(2)}: \chi^{(3)}$ model considered in this work, this means that one term in the Lagrangian should not be varied when deriving the equation for the fundamental wave. We demonstrate that the VA can be applied to such semi-Lagrangian systems as efficiently as to their full Lagrangian counterparts. Second, we have shown that, by the addition of an infinitesimal tail, which does not vanish at infinity, to the usual soliton ansatz, and demanding, after performing the variation, that the amplitude of the tail be zero, we obtain an approximate analytical criterion for locating embedded solitons inside a family of delocalized ones, i.e., isolated truly localized solutions existing inside the continuous spectrum of radiation modes. The criterion takes the form of orthogonality between the radiation mode contained in the infinitesimal tail and the core of the delocalized soliton. To test the criterion, we have applied it to both the semi-Lagrangian truncated version of the $\chi^{(2)}: \chi^{(3)}$ model and to the same model in its full form. In the former model, the criterion, combined with the VA for the soliton proper, yields a result which completely coincides with the previously found exact solution for the embedded soliton. In the latter model, the criterion predicts the wavenumber corresponding to the embedded soliton with a relative error $\approx 1 \%$.

\section{Acknowledgement}

B.A.M. appreciates hospitality of the Department of Mathematics at the University of Central Florida. This research has been supported in part by NSF grant \# DMS0129714.

\section{References}

[1] A.C. Newell, J. Math. Phys. 18 (1977) 922; 19 (1978) 1126; Rocky Mount. J. Math. 8 (1979) 25.

[2] D.J. Kaup and A.C. Newell, Phys. Rev. B 18 (1978) 5162 (1978); Proc. Roy. Soc. L. A 361 (1978) 413 (1978).

[3] J.P. Boyd. Weakly Nonlocal Solitary Waves and Beyond-All-Orders Asymptotics (Kluwer: Dodrecht, Boston, and London, 1998).

[4] D.J. Kaup, T.I. Lakoba and B.A. Malomed, J. Opt. Soc. Am. B 14 (1997) 1199 (1997); D.J. Kaup and B.A. Malomed, ibid. B 15 (1998) 2838.

[5] R. Grimshaw and P. Cook, in Hydrodynamics, ed. by A.T. Chang, J.H. Lee, and D.Y.C. Leung (Balkema: Rotterdam, 1996); A.R. Champneys and M.D. Groves, J. Fluid Mech. 342 (1997) 199. 
[6] A.R. Champneys, B.A. Malomed, J. Yang, and D.J. Kaup, Physica D 152-153 (2001) 340.

[7] J. Yang, B.A. Malomed, and D.J. Kaup, Phys. Rev. Lett. 83 (1999) 1958.

[8] B.A. Malomed, Progress in Optics 43 (2002) 69.

[9] S. Trillo, A.V. Buryak, and Y.S. Kivshar, Opt. Commun. 122 (1996) 200; O. Bang, Y.S. Kivshar, and A.V. Buryak, Opt. Lett. 22 (1997) 1680.

[10] L. Torner, IEEE Phot. Tech. Lett. 11 (1999) 1268); O. Bang, C. Clausen, P. Christiansen, and L. Torner, Opt. Lett. 24 (1999) 1413.

\section{Figure Captions}

Fig. 1. A comparison between the numerical shape (solid curves) of an ordinary (non-embedded) soliton of the semi-Lagrangian (truncated) system of equations (10) and (11), as found by the shooting method, and the result (dashed curves) obtained from the modified variational approximation based on Eqs. (13), (15), and (16). The parameters are $q=1, \delta=1, \gamma_{1}=0$, and $\gamma_{2}=-1 / 4$, and both the :

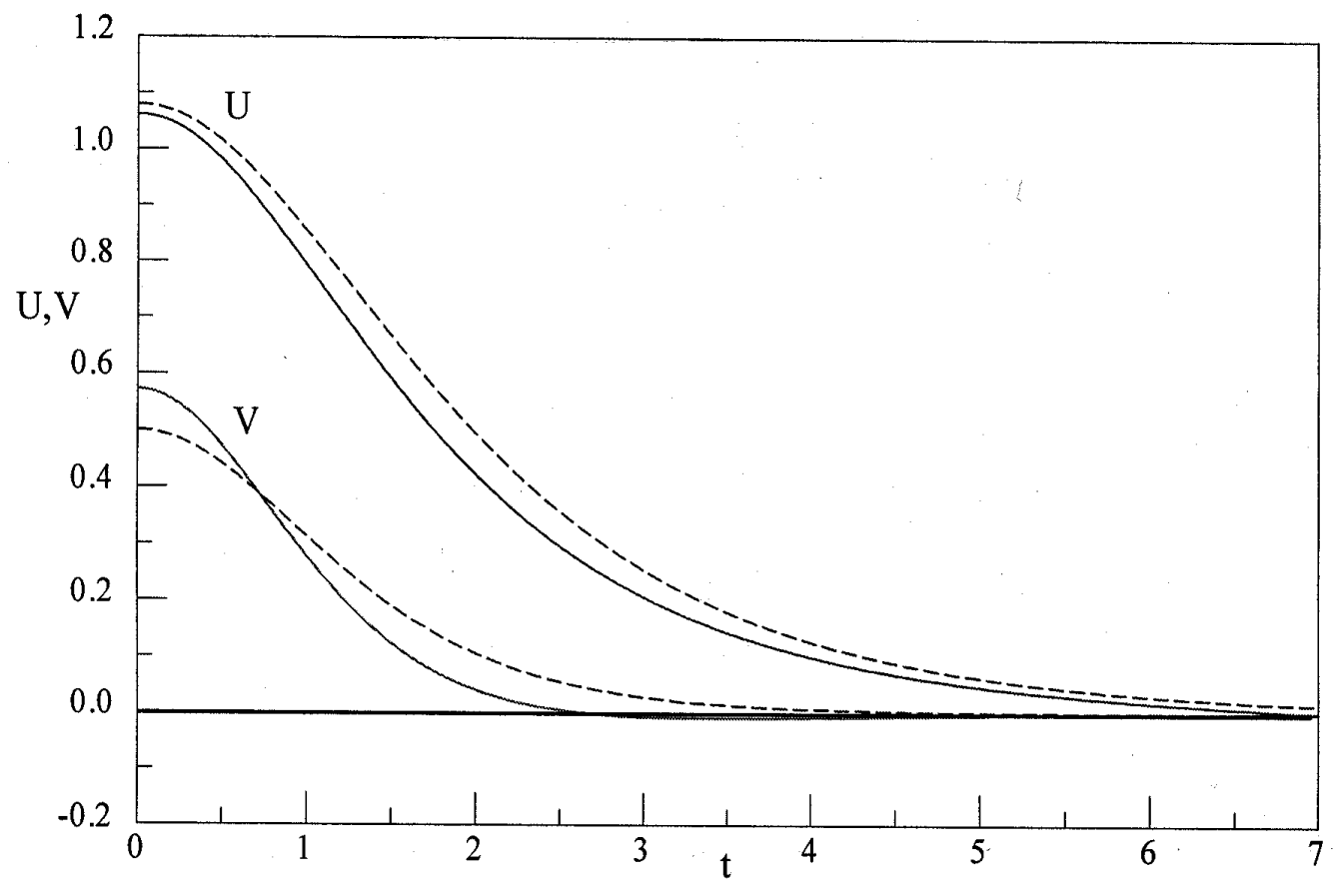

